\title{
CROSS-STREAM DEPENDENCY MODELING FOR HMM-BASED SPEECH SYNTHESIS
}

\author{
Zhen-Hua Ling ${ }^{1}$, Wei Zhang ${ }^{2}$,Ren-Hua Wang ${ }^{1}$ \\ ${ }^{1}$ iFlytek Research, Hefei \\ ${ }^{2}$ Department of Computer Science, Ocean University of China, Qingdao \\ zhling@iflytek.com, weizhang@ouc.edu.cn, rhwang@iflytek.com
}

\begin{abstract}
This paper presents a method that the dependency between F0 and spectral features are modeled for the HMM-based parametric speech synthesis system. In conventional systems these two features are modeled as two independent streams, which is inconsistent with the fact that there always exists interaction between the extracted $\mathrm{F} 0$ and spectral parameters for model training. A piecewise linear transform is introduced in this paper to explicitly model the dependency of spectrum on F0. The results of our experiments show that the proposed method is able to improve the accuracy of spectral parameter prediction if the $\mathrm{F} 0$ features are predicted based on a reliable voicing decision.
\end{abstract}

Index Terms - speech synthesis, hidden Markov model, STRAIGHT, cross-stream dependency, linear transform

\section{INTRODUCTION}

The HMM-based parametric speech synthesis method has been proposed and made significant progress in recent years [1]. In this method, spectrum, F0, and duration are modeled simultaneously in a unified framework of HMMs. During synthesis, the parameters are generated from HMMs [2] and then sent to a parametric synthesizer to reconstruct a speech waveform.

Conventionally, a multi-stream model structure is adopted for unified F0 and spectrum modeling. In this model structure, the two feature streams share the same state sequence and are assumed to be independent with each other once the state sequence is given. However, this assumption conflicts with the fact that there always exists dependency between the extracted F0 and spectrum for model training. Such dependency is caused by not only the physiological constraint of speech production but also the limitations of the speech analysis methods. For example, STRAIGHT [3] as a highperformance speech vocoder is commonly used in current systems $[4,5]$, which is able to remove the time-domain and frequency-domain periodicity from the speech spectrum. But the spectral envelope extracted by STRAIGHT still consists

This work was partially supported by National Natural Science Foundation of China (Grant No.: 60602017). of F0-dependent component. In order to explain this point, let us have a look at the production procedure of speech signal $s(t)$ at first.

$$
s(t)=e(t) * g(t) * v(t)
$$

where $e(t), g(t)$, and $v(t)$ denote pulse train, glottal waveform and impulse response of vocal tract filter respectively; * means convolution. The corresponding frequency-domain representation is

$$
S(\omega)=E(\omega) \cdot G(\omega) \cdot V(\omega) .
$$

STRAIGHT algorithm decomposes speech signal into an excitation component and a smoothed spectral envelope [3]. The excitation signal is designed as a pulse train with some phase manipulation, which is determined by F0 only and similar to $E(\omega)$ in Eq.(2). Therefore, the extracted spectrum corresponds to $G(\omega) \cdot V(\omega)$, which is a product of the glottal spectrum and the frequency response of vocal tract filter. Some previous researches on the characteristics of glottal waveform demonstrate that the shape of glottal spectrum $G(\omega)$ is dependent on the concurrent F0, especially the position of "glottal formant", which is directly proportional to the F0 [6]. Hence, the extracted spectral envelope by STRAIGHT consists of F0-dependent component, which is inconsistent with the feature-independent assumption. In order to solve this problem, a cross-stream dependency modeling method is proposed in this paper. A piecewise linear transform is introduced into model training and parameter generation to explicitly present the dependency of spectral features on F0.

This paper is organized as follows. In Section 2, the details of proposed method is introduced after a brief description of the baseline system. Section 3 gives the experiment results and Section 4 is the conclusion.

\section{METHOD}

\subsection{Baseline system}

The flowchart of HMM-based parametric speech synthesis system is shown in Fig.1. At the training stage, the F0 and $D$-dimension spectral parameters are extracted from the waveforms of training database. Then a set of HMMs $\lambda$ for 


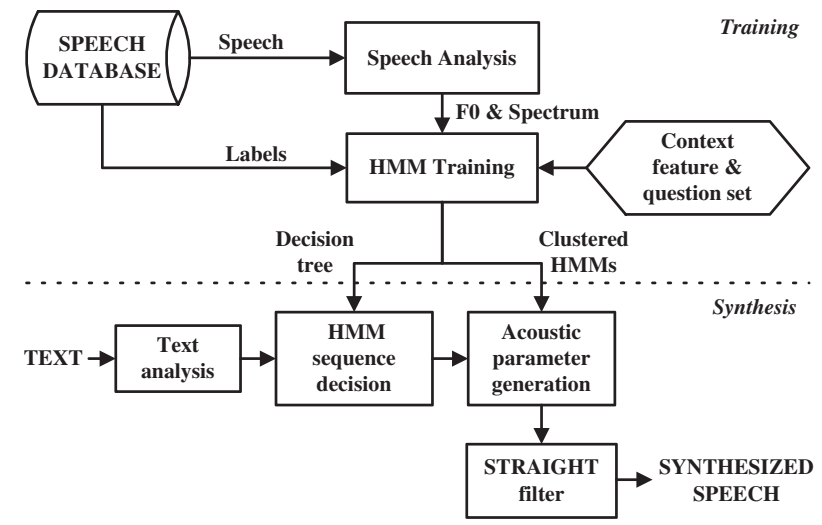

Fig. 1. Flowchart of HMM-based parametric speech synthesis system.

context-dependent phonemes are estimated to maximize the likelihood function $P(\boldsymbol{X}, \boldsymbol{Y} \mid \lambda)$ for the training data. Here $\boldsymbol{X}=\left[\boldsymbol{x}_{1}^{\top}, \boldsymbol{x}_{2}^{\top}, \ldots, \boldsymbol{x}_{N}^{\top}\right]^{\top}$ is the observed spectral feature sequence and $\boldsymbol{Y}=\left(\boldsymbol{y}_{1}, \boldsymbol{y}_{2}, \ldots, \boldsymbol{y}_{N}\right)$ is the observed F0 feature sequence; $(\cdot)^{\top}$ means the matrix transpose and $N$ is the length of two sequences. For each frame, the spectral feature vector $\boldsymbol{x}_{t}=\left[\boldsymbol{x}_{S_{t}}^{\boldsymbol{\top}}, \Delta \boldsymbol{x}_{S_{t}}^{\top}, \Delta^{2} \boldsymbol{x}_{S_{t}}^{\boldsymbol{\top}}\right]^{\top} \in \mathcal{R}^{3 D}$ is composed of static spectral parameters $\boldsymbol{x}_{S_{t}} \in \mathcal{R}^{D}$ and their velocity and acceleration components. The F0 features are modeled by a Multi-Space probability Distribution HMM (MSD-HMM) [7]. For each frame, $\boldsymbol{y}_{t}=\left(s_{t}, \boldsymbol{v}_{t}\right)$, where $s_{t}$ denotes the space index of $\boldsymbol{y}_{t}$ and $\boldsymbol{v}_{t}$ denotes the value of $\boldsymbol{y}_{t}$ in space $s_{t}$. Here we have $s_{t} \in\{0,1\}$, where 0 and 1 present voiced space and unvoiced space respectively. In voiced space, $\boldsymbol{v}_{t}=\left[\boldsymbol{v}_{S_{t}}^{\top}, \Delta \boldsymbol{v}_{S_{t}}^{\top}, \Delta^{2} \boldsymbol{v}_{S_{t}}^{\top}\right]^{\top} \in \mathcal{R}^{3}$ is composed of static F0 parameter $\boldsymbol{v}_{S_{t}} \in \mathcal{R}^{1}$ and their velocity and acceleration components; in unvoiced space, $v_{t} \in \mathcal{R}^{0}$.

In the baseline system, a two-stream model structure is used for unified F0 and spectrum modeling. ${ }^{1}$ In this model, F0 and spectral features are assumed to be independent given state sequence. Therefore, we have

$$
\begin{array}{r}
P(\boldsymbol{X}, \boldsymbol{Y} \mid \lambda)=\sum_{\forall \boldsymbol{q}} P(\boldsymbol{X}, \boldsymbol{Y}, \boldsymbol{q} \mid \lambda) \\
=\sum_{\forall \boldsymbol{q}} \pi_{q_{0}} \prod_{t=1}^{N} a_{q_{t-1} q_{t}} b\left(\boldsymbol{x}_{t}, \boldsymbol{y}_{t}\right) \\
b_{j}\left(\boldsymbol{x}_{t}, \boldsymbol{y}_{t}\right)=b_{j}\left(\boldsymbol{x}_{t}\right) b_{j}\left(\boldsymbol{y}_{t}\right) \\
b_{j}\left(\boldsymbol{x}_{t}\right)=\mathcal{N}\left(\boldsymbol{x}_{t} ; \boldsymbol{\mu}_{\boldsymbol{X}_{j}}, \boldsymbol{\Sigma}_{\boldsymbol{X}_{j}}\right) \\
b_{j}\left(\boldsymbol{y}_{t}\right)=w_{\boldsymbol{Y}_{j}}^{\left(s_{t}\right)} \mathcal{N}\left(\boldsymbol{v}_{t} ; \boldsymbol{\mu}_{\boldsymbol{Y}_{j}}^{\left(s_{t}\right)}, \boldsymbol{\Sigma}_{\boldsymbol{Y}_{j}}^{\left(s_{t}\right)}\right)
\end{array}
$$

where $\boldsymbol{q}=\left\{q_{1}, q_{2}, \ldots, q_{N}\right\}$ denotes the state sequence shared by spectral and F0 features; $\pi_{j}$ and $a_{i j}$ represent initial state

\footnotetext{
${ }^{1}$ In practical implementation, a four-stream model structure is adopted considering the space indexes of static F0 and dynamic F0 may be different. Here, we assume a unique space index is shared by all F0 features of each frame and use a two-stream model structure to simplify the explanation.
}

probability and state transit probability; $b_{j}(\cdot)$ means the state observation probability density function (PDF) for state $j$; $\mathcal{N}(; \boldsymbol{\mu}, \boldsymbol{\Sigma})$ represents a Gaussian distribution with a mean vector $\boldsymbol{\mu}$ and a covariance matrix $\boldsymbol{\Sigma} ; w_{\boldsymbol{Y}_{j}}^{\left(s_{t}\right)}$ is the probability of space $s_{t}$ for state $j$. Because $\boldsymbol{v}_{t} \in \mathcal{R}^{0}$ when $s_{t}=1$ and $w_{\boldsymbol{Y}_{j}}^{(0)}+w_{\boldsymbol{Y}_{j}}^{(1)} \equiv 1$, it is not necessary to estimate the PDF for unvoiced space of F0 features. Finally, the model parameter set is $\left\{a_{i j}, \boldsymbol{\mu}_{\boldsymbol{X}_{j}}, \boldsymbol{\Sigma}_{\boldsymbol{X}_{j}}, w_{\boldsymbol{Y}_{j}}^{(0)}, \boldsymbol{\mu}_{\boldsymbol{Y}_{j}}^{(0)}, \boldsymbol{\Sigma}_{\boldsymbol{Y}_{j}}^{(0)}\right\}$, which can be estimated by EM algorithm described in [7]. A decisiontree-based model clustering technique [1] is applied after the training for context-dependent HMMs to deal with datasparsity problems and to avoid over-fitting to the training data. Then, we take the state alignment results using trained HMMs and use them to train contextually dependent state duration probabilities [8].

During synthesis, the result of the text analysis is used to decide the sentence HMM according to the clustering decision tree. Then, the Maximum-Likelihood Parameter Generation (MLPG) algorithm using dynamic features [2] is applied to generate the optimal F0 and spectral parameters for each frame. In the baseline system, these two features are generated independently.

\subsection{Cross-stream dependency modeling}

In order to present the interaction between the F0 and spectral parameters, a cross-stream dependency modeling method is proposed here. In this method, the generation of spectral features is not only determined by the model parameters of HMM states, but also dependent on the concurrent F0 features. Thus, Eq.(4) can be rewritten as

$$
b_{j}\left(\boldsymbol{x}_{t}, \boldsymbol{y}_{t}\right)=b_{j}\left(\boldsymbol{x}_{t} \mid \boldsymbol{y}_{t}\right) b_{j}\left(\boldsymbol{y}_{t}\right) .
$$

In order to facilitate the parameter estimation, a piecewise linear transform is used to present the conditional probability of spectral features on $\mathrm{F} 0$ features as

$$
b_{j}\left(\boldsymbol{x}_{t} \mid \boldsymbol{y}_{t}\right)=\mathcal{N}\left(\boldsymbol{x}_{t} ; \boldsymbol{A}_{j}^{\left(s_{t}\right)} \boldsymbol{v}_{t}+\boldsymbol{\mu}_{\boldsymbol{X}_{j}}, \boldsymbol{\Sigma}_{\boldsymbol{X}_{j}}\right)
$$

where $\boldsymbol{A}_{j}^{\left(s_{t}\right)}$ denotes the transform matrix when frame $t$ belongs to state $j$ and current F0 belongs to space $s_{t}$. The transform matrix is state-dependent, so a globally piecewise linear transform can be achieved. Because $\boldsymbol{v}_{t} \in \mathcal{R}^{0}$ for unvoiced frames, we define $\boldsymbol{\mu}_{\mathbf{X}_{j}}^{(1)}=\boldsymbol{A}_{j}^{(1)} \boldsymbol{v}_{t}$ and estimate $\boldsymbol{\mu}_{\mathbf{X}_{j}}^{(1)}$ instead of $\boldsymbol{A}_{j}^{(1)} \boldsymbol{v}_{t}$. The re-estimation formulas can be derived using EM algorithm as

$$
\begin{aligned}
\boldsymbol{A}_{j}^{(0)^{\prime}}= & {\left[\sum_{t \in T(\boldsymbol{Y}, 0)} \gamma_{j}(t)\left(\boldsymbol{x}_{t}-\boldsymbol{\mu}_{\boldsymbol{X}_{j}}\right) \boldsymbol{v}_{t}^{\top}\right] } \\
& \cdot\left[\sum_{t \in T(\boldsymbol{Y}, 0)} \gamma_{j}(t) \boldsymbol{v}_{t} \boldsymbol{v}_{t}^{\top}\right]^{-1}
\end{aligned}
$$




$$
\begin{gathered}
\boldsymbol{\mu}_{\boldsymbol{X}_{j}}^{(1)^{\prime}}=\frac{\sum_{t \in T(\boldsymbol{Y}, 1)} \gamma_{j}(t)\left(\boldsymbol{x}_{t}-\boldsymbol{\mu}_{\boldsymbol{X}_{j}}\right)}{\sum_{t \in T(\boldsymbol{Y}, 1)} \gamma_{j}(t)} \\
T(\boldsymbol{Y}, g)=\left\{t \mid s_{t}=g\right\}, g \in\{0,1\} \\
\boldsymbol{\mu}_{\boldsymbol{X}_{j}}^{\prime}=\frac{\sum_{t=1}^{T} \gamma_{j}(t)\left(\boldsymbol{x}_{t}-\boldsymbol{A}_{j}^{\left(s_{t}\right)^{\prime}} \boldsymbol{v}_{t}\right)}{\sum_{t=1}^{T} \gamma_{j}(t)} \\
\boldsymbol{\Sigma}_{\boldsymbol{X}_{j}}^{\prime}=\frac{1}{\sum_{t=1}^{T} \gamma_{j}(t)} \cdot \\
{\left[\sum_{t=1}^{T} \gamma_{j}(t)\left(\boldsymbol{x}_{t}-\boldsymbol{A}_{j}^{\left(s_{t}\right)^{\prime}} \boldsymbol{v}_{t}-\boldsymbol{\mu}_{\boldsymbol{X}_{j}}^{\prime}\right)\left(\boldsymbol{x}_{t}-\boldsymbol{A}_{j}^{\left(s_{t}\right)^{\prime}} \boldsymbol{v}_{t}-\boldsymbol{\mu}_{\boldsymbol{X}_{j}}^{\prime}\right)^{T}\right]}
\end{gathered}
$$

where $\gamma_{j}(t)$ is the state occupancy probability of frame $t$ belonging to state $j$. Model parameters $\left\{a_{i j}, \boldsymbol{\mu}_{\boldsymbol{X}_{j}}, \boldsymbol{\Sigma}_{\boldsymbol{X}_{j}}, w_{\boldsymbol{Y}_{j}}^{(0)}\right.$, $\left.\boldsymbol{\mu}_{\boldsymbol{Y}_{j}}^{(0)}, \boldsymbol{\Sigma}_{\boldsymbol{Y}_{j}}^{(0)}\right\}$ given by the baseline system are used as initial parameters for iterative re-estimation. $\boldsymbol{A}_{j}^{(0)}$ and $\boldsymbol{\mu}_{\boldsymbol{X}_{j}}^{(1)}$ are set to be zero for the first iteration.

At the synthesis stage, the same maximum likelihood criterion is followed to generate the optimal static spectral feature sequence $\boldsymbol{X}_{S}^{*}$ and F0 feature sequence $\boldsymbol{Y}_{S}^{*}$. Here, the F0 and spectral parameters are not generated independently. The dependency between these two features needs to be considered in the generation procedure. We have

$$
\begin{aligned}
& \left(\boldsymbol{X}_{S}^{*}, \boldsymbol{Y}_{S}^{*}\right)=\arg \max _{\boldsymbol{X}_{S}, \boldsymbol{Y}_{S}} P(\boldsymbol{X}, \boldsymbol{Y} \mid \lambda) \\
& =\arg \max _{\boldsymbol{X}_{S}, \boldsymbol{Y}_{S}} \sum_{\forall \boldsymbol{q}} P\left(\boldsymbol{W}_{\boldsymbol{X}} \boldsymbol{X}_{S}, \boldsymbol{W}_{\boldsymbol{Y}} \boldsymbol{Y}_{S}, \boldsymbol{q} \mid \lambda\right) \\
& \simeq \arg \max _{\boldsymbol{X}_{S}, \boldsymbol{Y}_{S}} \max _{\mathbf{q}} P\left(\boldsymbol{W}_{\boldsymbol{X}} \boldsymbol{X}_{S}, \boldsymbol{W}_{\boldsymbol{Y}} \boldsymbol{Y}_{S}, \boldsymbol{q} \mid \lambda\right) \\
& =\arg \max _{\boldsymbol{X}_{S}, \boldsymbol{Y}_{S}} \max _{\mathbf{q}} P\left(\mathbf{W}_{\boldsymbol{X}} \boldsymbol{X}_{S}{ }^{\prime} \boldsymbol{W}_{\boldsymbol{Y}} \boldsymbol{Y}_{S}, \boldsymbol{q}, \lambda\right) P\left(\boldsymbol{W}_{\boldsymbol{Y}} \boldsymbol{Y}_{S} \mid \boldsymbol{q}, \lambda\right) P(\boldsymbol{q} \mid \lambda)
\end{aligned}
$$

where $\boldsymbol{X}_{S}=\left[\boldsymbol{x}_{S_{1}}^{\top}, \boldsymbol{x}_{S_{2}}^{\top}, \ldots, \boldsymbol{x}_{S_{N}}^{\top}\right]^{\top}, \boldsymbol{Y}_{S}=\left(\boldsymbol{y}_{S_{1}}, \boldsymbol{y}_{S_{2}}, \ldots, \boldsymbol{y}_{S_{N}}\right)$, $\boldsymbol{y}_{S_{t}}=\left(s_{t}, \boldsymbol{v}_{S_{t}}\right) ; \boldsymbol{X}=\boldsymbol{W}_{\boldsymbol{X}} \boldsymbol{X}_{S}, \boldsymbol{Y}=\boldsymbol{W}_{\boldsymbol{Y}} \boldsymbol{Y}_{S},{ }^{2} \boldsymbol{W}_{\boldsymbol{X}}$ and $\boldsymbol{W}_{\boldsymbol{Y}}$ are the matric that are used to calculate the complete feature sequences based on static parameters [2]. The solution of Eq.(14) can be approximated by three steps:

1) Generate the optimal state sequence $\boldsymbol{q}^{*}$ using duration model [8] as

$$
\boldsymbol{q}^{*}=\arg \max _{\boldsymbol{q}} P(\boldsymbol{q} \mid \lambda)
$$

2) Generate the optimal static F0 feature sequence $\boldsymbol{Y}_{S}^{*}$ as

$$
\boldsymbol{Y}_{S}^{*}=\arg \max _{\boldsymbol{Y}_{S}} P\left(\boldsymbol{W}_{\boldsymbol{Y}} \boldsymbol{Y}_{S} \mid \boldsymbol{q}, \lambda\right) .
$$

At first, the space index $s_{t}$ for each frame is predicted according to space probability $w_{\boldsymbol{Y}_{j}}^{(0)}$ and $w_{\boldsymbol{Y}_{j}}^{(1)}$. After the voicing decision, the MLPG algorithm [2] is applied to generate the optimal F0 parameters.

\footnotetext{
${ }^{2}$ In fact, the transform from static F0 parameters to complete F0 feature sequence can not be presented by the linear transform matrix $\boldsymbol{W}_{\boldsymbol{Y}}$ considering the multi-space property of F0. Here, we use $\boldsymbol{W}_{\boldsymbol{Y}}$ to simplify the explanation.
}

3) Bring the generated optimal F0 features into Eq.(8) and get the state PDFs for spectral features. Finally the optimal spectral feature sequence $\boldsymbol{X}_{S}^{*}$ can be generated as

$$
\boldsymbol{X}_{S}^{*}=\arg \max _{\boldsymbol{X}_{S}} P\left(\mathbf{W}_{\boldsymbol{X}} \boldsymbol{X}_{S} \mid \boldsymbol{W}_{\boldsymbol{Y}} \boldsymbol{Y}_{S}^{*}, \boldsymbol{q}, \lambda\right) .
$$

This can also be solved by the MLPG algorithm [2].

\section{EXPERIMENTS}

\subsection{System construction}

The database used for experiments consists of 1,000 phonetically balanced Chinese sentences pronounced by a female speaker. 900 sentences are selected randomly from the database for system training and the remaining 100 sentences are used as test set. 40th order frequency warped LSFs and an extra gain dimension were derived from the spectral envelope provided by STRAIGHT analysis and were used as spectral parameters. 5-state left-to-right with no skip HMM structure was adopted for each initial/final in Chinese. Context features and question set for decision tree clustering were designed considering the characteristics of Chinese. Then the baseline system and the proposed system with cross-stream dependency modeling were constructed following the instructions in section 2.1 and section 2.2 respectively. In the training of proposed system, transform matrix $\boldsymbol{A}_{j}^{\left(s_{t}\right)}$ was set to be statedependent and was tied using the clustering decision tree for spectral models.

\subsection{Objective evaluation}

In order to evaluate the effectiveness of proposed method, an objective measure is used by calculating the Root Mean Square Error (RMSE) of the predicted LSF sequences compared with the natural ones. The calculation for two LSF sequences $\boldsymbol{L}=\left[\boldsymbol{l}_{1}, \boldsymbol{l}_{2}, \ldots, \boldsymbol{l}_{N}\right]$ and $\tilde{\boldsymbol{L}}=\left[\tilde{\boldsymbol{l}}_{1}, \tilde{\boldsymbol{l}}_{2}, \ldots, \tilde{\boldsymbol{l}}_{N}\right]$ is defined as

$$
\begin{aligned}
& \operatorname{RMSE}(\boldsymbol{L}, \tilde{\boldsymbol{L}})=\sqrt{\frac{1}{T} \sum_{t=1}^{T} \operatorname{Err}_{L S F}^{2}\left(\boldsymbol{l}_{t}, \tilde{\boldsymbol{l}}_{t}\right)} \\
& \operatorname{Err}_{L S F}\left(\boldsymbol{l}_{t}, \tilde{\boldsymbol{l}}_{t}\right)=\sqrt{\sum_{d=1}^{D}\left(l_{t d}-\tilde{l}_{t d}\right)^{2}} \\
& \boldsymbol{l}_{t}=\left[l_{t 1}, l_{t 2}, \ldots, l_{t D}\right]^{\top}, \tilde{\boldsymbol{l}}_{t}=\left[\tilde{l}_{t 1}, \tilde{l}_{t 2}, \ldots, \tilde{l}_{t D}\right]^{\top}
\end{aligned}
$$

where $N$ is the sequence length, $D$ is the dimension of LSF and function $\operatorname{Err}_{L S F}(\cdot)$ defines the Euclidean distance between two LSF vectors. Finally, the state durations of generated LSF sequences were set to be the same as the natural ones in order to simplify the calculation of RMSE in the following experiments.

The objective evaluation was conducted on the training set and the test set respectively. The results are shown in Fig.2. 


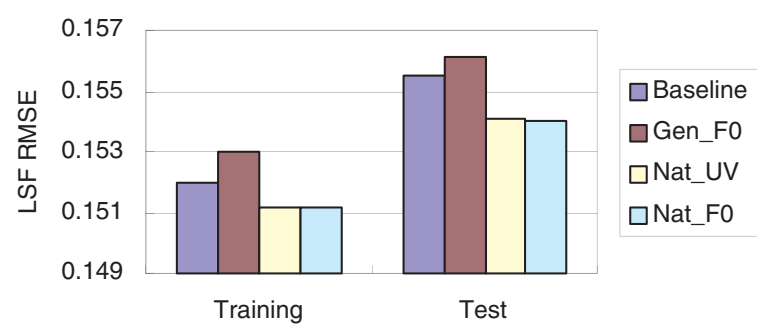

Fig. 2. Objective evaluation of LSF RMSE on the training set and the test set.

Three conditions are considered for spectral parameter generation in the proposed system, they are:

- Gen_F0: $\boldsymbol{Y}_{S}^{*}$ in Eq.(17) is generated from the trained models as Eq.(16).

- Nat_UV: Same as Gen_FO except that the space index $s_{t}$ for the $\mathrm{F} 0$ of each frame is not predicted but copied from the natural $\mathrm{F} 0$ features.

- Nat_FO: $\boldsymbol{Y}_{S}^{*}$ in Eq.(17) is completely copied from the natural F0 features.

The results of a T-test show that the differences between the prediction errors of the baseline system and the three conditions of proposed system are all significant at 0.05 significance level on both the training set and the test set. From Fig.2, we can see that:

1) The proposed method can decrease the RMSE of predicted LSFs on both training set and test set effectively if the natural F0 features are given (comparing Nat_FO with Baseline). This proves that the cross-stream dependency modeling can give a better description for the relationship between $\mathrm{F} 0$ and spectral features compared with the baseline system which assume these two features are independent.

2) If the natural F0 features are not given, the proposed method decreases the accuracy of LSF prediction (comparing Gen_FO with Baseline).

3) The prediction of F0 space index (i.e. voicing decision) plays an important role in the performance of proposed method. If the generated F0 and natural F0 have the same voicing decision for each frame, the RMSE of predicted LSFs can also get decreased significantly (comparing Nat_UV with Baseline).

Although some significant improvements can be achieved in the objective evaluation, an informal listening test shows that the subjective differences between the baseline system and the proposed systems are not significant.

\section{CONCLUSIONS}

This paper presents a cross-stream dependency modeling method for HMM-based parametric speech synthesis system. A piecewise linear transform is used to model the dependency explicitly. The results of our experiments proves that the proposed method is able to improve the accuracy of spectral parameter prediction effectively if the F0 features are predicted with a natural voicing decision. However, these are still some problems with the current method, such as the influence of voicing decision on the generation of spectral parameters and the insignificant subjective improvement. To find a better strategy to deal with the multi-space property of F0 in the cross-stream dependency modeling will be a task of our future work.

\section{REFERENCES}

[1] K. Tokuda, H. Zen, and A. W. Black, "HMM-based approach to multilingual speech synthesis," in Text to speech synthesis: New paradigms and advances, S. Narayanan and A. Alwan, Eds. Prentice Hall, 2004.

[2] K. Tokuda, T. kabayashi, and S. Imai, "Speech parameter generation from HMM using dynamic features," in ICASSP, 1995, pp. 660-663.

[3] H. Kawahara, I. Masuda-Katsuse, and A. de Cheveigne, "Restructuring speech representations using pitchadaptive time-frequency smoothing and an instantaneous-frequency-based F0 extraction: possible role of a repetitive structure in sounds," Speech Communication, vol. 27, pp. 187-207, 1999.

[4] H. Zen, T. Toda, M. Nakamura, and K. Tokuda, "Details of Nitech HMM-based speech synthesis system for the Blizzard Challenge 2005," IEICE Trans. Inf. and Syst., vol. E90-D, no. 1, pp. 325-333, 2007.

[5] Z. Ling, Y. Wu, Y. Wang, L. Qin, and R. Wang, "USTC system for Blizzard Challenge 2006: an improved HMMbased speech synthesis method," in Blizzard Challenge Workshop, 2006.

[6] C. d'Alessandro and B. Doval, "Voice quality modification for emotional speech synthesis," in Eurospeech, 2003, pp. 1653-1656.

[7] K. Tokuda, T. Masuko, N. Miyazaki, and T. Kobayashi, "Hidden Markov models based on multi-space probability distribution for pitch pattern modeling," in ICASSP, 1999, pp. 229-232.

[8] T. Yoshimura, K. Tokuda, T. Masuko, T. Kobayashi, and T. Kitamura, "Duration modeling in HMM-based speech synthesis system," in ICSLP, 1998, vol. 2, pp. 29-32. 